\title{
Paclitaxel resistance is associated with switch from apoptotic to autophagic cell death in MCF-7 breast cancer cells
}

\author{
GMA Ajabnoor ${ }^{1,2}$, T Crook $^{3}$ and HM Coley ${ }^{\star, 2}$
}

Taxanes remain first line chemotherapy in management of metastatic breast cancer and have a key role in epithelial ovarian cancer, with increasingly common use of weekly paclitaxel dosing regimens. However, their clinical utility is limited by the development of chemoresistance. To address this, we modelled in vitro paclitaxel resistance in MCF-7 cells. We show that at clinically relevant drug doses, emerging paclitaxel resistance is associated with profound changes in cell death responses and a switch from apoptosis to autophagy as the principal mechanism of drug-induced cytotoxicity. This was characterised by a complete absence of caspase-mediated apoptotic cell death (using the pan-caspase-inhibitor Z-VAD) in paclitaxel-resistant MCF-7TaxR cells, compared with parent MCF-7 or MDA-MB-231 cell lines on paclitaxel challenge, downregulation of caspase-7, caspase-9 and BCl2-interacting mediator of cell death (BIM) expression. Silencing with small interfering RNA to BIM in MCF-7 parental cells was sufficient to confer paclitaxel resistance, inferring the significance in downregulation of this protein in contributing to the resistant phenotype of the MCF-7TaxR cell line. Conversely, there was an increased autophagic response in the MCF-7TaxR cell line with reduced phospho-mTOR and relative resistance to the mTOR inhibitors rapamycin and RAD001. In conclusion, we show for the first time that paclitaxel resistance is associated with profound changes in cell death response with deletion of multiple apoptotic factors balanced by upregulation of the autophagic pathway and collateral sensitivity to platinum. Cell Death and Disease (2012) 3, e260; doi:10.1038/cddis.2011.139; published online 26 January 2012

Subject Category: Experimental Medicine

Despite significant improvements in clinical outcomes, metastatic breast cancer (MBC) remains an essentially incurable disease. The major factor limiting long-term control of $M B C$ is development of chemotherapy resistance. Significant advances in understanding of some mechanisms of anti-cancer drug resistance have not been paralleled by introduction of novel strategies to circumvent or avoid resistance in clinical practice. A convincing body of experimental evidence implies that defective apoptosis may be an important mechanism underlying chemoresistance. ${ }^{1-3}$

Here, we have examined the evolution of paclitaxel resistance in an MCF-7 cell line,MCF-7TaxR, grown in the presence of paclitaxel for a limited time period. We have previously published data regarding the cross-resistance profile of the MCF-7TaxR line ${ }^{4}$ that described upregulation of $A B C B 1$, but little cross-resistance to the natural product drugs etoposide, doxorubicin and colchicine. In MCF-7TaxR cells, we did not see any changes in the expression levels of beta-III tubulin compared with parental MCF-7 cells (data not shown), another mechanism cited for paclitaxel resistance. ${ }^{5}$ Initial analyses indicated a marked decrease in the apoptotic response of paclitaxel-resistant MCF-7 cells to a range of agents. However, attenuation of apoptosis seen in MCF-7TaxR cells did not coincide with resistance to many of those agents. Our investigation set out to explore the underlying basis for these changes. Here, we report that the reduction of apoptosis occurring upon acquisition of paclitaxel resistance is balanced by upregulation of autophagy as the principal mechanism of cytotoxicity and cell death.

\section{Results}

Paclitaxel-resistant MCF-7 cells show an unusual spectrum of cross-resistance in the presence of P-glycoprotein. We tested whether a multidrug resistant (MDR) phenotype was present by challenging the cells with doxorubicin and an irrelevant cytotoxic drug cisplatin (Figure 1b). During the development of paclitaxel resistance, MCF-7

\footnotetext{
${ }^{1}$ Clinical Biochemistry Department, Faculty of Medicine, King Abdulaziz University, P.O Box 80200, Jeddah 21589, Kingdom of Saudi Arabia; ${ }^{2}$ Faculty of Health and Medical Sciences, University of Surrey, Guildford, Surrey GU2 7XH, UK and ${ }^{3}$ Medical Oncology, Charing Cross Hospital, London W6 8RF, UK

${ }^{*}$ Corresponding author: HM Coley, Biosciences Division, Faculty of Health and Medical Science, University of Surrey, Room 14AY04, Guildford, Surrey GU2 7XH, UK. Tel: + 441483 688617; Fax: + 441483686401 ; E-mail: h.coley@surrey.ac.uk

Keywords: breast cancer; caspase deletion; drug resistance; autophagic cell death

Abbreviations: $\mathrm{ABCB} 1, \mathrm{ATP}$-binding cassette protein $\mathrm{B} 1$; AKT, protein kinase B; BAD, BCl2-associated death promoter; BCL2, B-cell lymphoma-2; BIM, BCl2interacting mediator of cell death; DMSO, dimethyl sulphoxide; FAS, apoptosis-stimulating fragment; FBS, foetal bovine serum; FITC, fluoroscein-isothiocyanate; GAPDH, glyceraldehyde phosphate dehydrogenase; IAP, inhibitor of apoptosis; $I \mathrm{I}_{50}$, inhibitory concentration causing $50 \%$ loss of cell viability; $L C-3 \mid / I$, microtubuleassociated protein 1-light chain $3 \mathrm{l} / \mathrm{ll}$; MBC, metastatic breast cancer; MDR, multidrug resistant; mRNA, messenger ribonucleic acid; mTOR, mammalian target of rapamycin; MTT, 3-(4,5-dimethylthylthiazol-2-yl)-2,5-diphenyltetrazolium bromide; PI, propidium iodide; PVDF, polyvinylidene difluoride; qPCR, quantitiave polymerase chain reaction; SDS-PAGE, sodium dodecyl sulphate-polyacrylamide gel electrophoresis; SiRNA, small interfering RNA; Z-VAD(fmk), Z-Asp-Glu-Val-Asp chloromethylketone

Received 10.6.11; revised 29.11.11; accepted 01.12.11; Edited by A Stephanou
} 
cells showed a gradual upregulation of the MDR transporter P-glycoprotein (first evident at passage \#23 when paclitaxel resistance was established (Figures $1 \mathrm{~b}$ and $2 \mathrm{a}$ ). At this point in spite of upregulation of MDR1, a multidrug-resistant profile was not evident in the MCF-7TaxR cells from passage 23 onwards as only a modest increase in doxorubicin resistance was apparent and resistance to other MDR-associated agents, including etoposide was not seen. ${ }^{4}$ Interestingly, we saw a collateral sensitivity to cisplatin (Figure 1b) and to carboplatin (data not shown).

Loss of caspase-7 and -9 is associated with emerging paclitaxel resistance. As part of the characterisation of the resistant cell line MCF-7TaxR, we examined the role of apoptotic cell death response. As part of this analysis, we assessed expression of caspase-7 and -9 in MCF-7 cells with increasing resistance to paclitaxel. Downregulation of protein expression was first evident at passage 18 when cells were establishing resistance at a dose of $6 \mathrm{nM}$ paclitaxel and the messenger ribonucleic acid (mRNA) levels of both genes was undetectable from passage 23 onwards (Figures 2a and b). Loss of expression of caspases-7 and -9 was a stable molecular event as the MCF-7TaxR(-) variant line failed to show re-expression of either gene following several months of culture in the absence of paclitaxel.

Development of a paclitaxel-resistant MCF-7 cell line gives rise to marked changes in pro-and anti-apoptotic factors. Next, we analysed MCF-7TaxR cells for expression of pro-and anti-apoptotic proteins. There was a striking reduction in expression of $\mathrm{BIM}$ and $\mathrm{BCl} 2$ between passages 18-23, but levels of $\mathrm{BCl} 2$-associated death promoter were stable (Figure 2a).
Absence of BIM as a factor contributing to paclitaxel resistance. To evaluate a potential role for loss of BIM underlying the development of paclitaxel resistance, we used SiRNA to selectively block BIM expression in MCF-7 parental cells. SiRNA produced efficient downregulation of BIM and on subsequent challenge with paclitaxel a reduced apoptotic response was seen (Figure 2c). A series of repeat experiments showed that at $48 \mathrm{~h}$ viability in control (nonsilenced) cells following paclitaxel treatment $(20 \mathrm{nM})$ were $65.3 \%$ (relative to the viable population of the respective control). The corresponding data for the BIM silenced population was $85.8 \%$. As indicated in the figure legend, these data were shown to be statistically different from each other. Data indicate that if taken as a factor in isolation, deletion of BIM is sufficient to confer resistance to paclitaxel in MCF-7 cells.

Paclitaxel-resistant MCF-7 cells show loss of caspasemediated cell death. We tested for the ability of apoptotic induction in paclitaxel-resistant MCF-7TaxR by challenging cells with staurosporine (an agent frequently used to demonstrate apoptosis), paclitaxel and cisplatin in the absence or presence of the pan-caspase inhibitor Z-VAD using the Annexin $\mathrm{V}$ assay with flow cytometric analysis. Figure $3 A$ indicates the extent of protection afforded by Z-VAD pre-treatment of cells to a dose of staurosporine. Figure 3Acindicates the induction of cell death (FITC and PI-positive populations) and the protective effect afforded by Z-VAD (Figure 3Ad). Figure 3B indicates the extent of Z-VAD protection from caspase-mediated staurosporineinduced cell death in three breast cancer cell lines $(n=4)$ based on the size of the viable cell population (lower lefthand quadrant of panels Figures $3 \mathrm{~A} \mathrm{C}$ and $\mathrm{d})$. Overall, the involvement of caspase-mediated cell death following

\begin{tabular}{|l|l|l|l|l|l|l|l|l|l|l|l|l|l|l|l|l|l|l|l|l|l|l|l|}
\hline \begin{tabular}{|} 
PASSAGE \\
$\#$
\end{tabular} & 1 & 2 & 3 & 4 & 5 & 6 & 7 & 8 & 9 & 10 & 11 & 12 & 13 & 14 & 15 & 16 & 17 & 18 & 19 & 20 & 21 & 22 & 23 \\
\hline WEEK & 1 & & 2 & 3 & & 4 & & 5 & 6 & 7 & & & 8 & & 9 & & & 10 & & 11 & & 12 & \\
\hline $\begin{array}{c}\text { PACLITAXEL } \\
\text { DOSE nM }\end{array}$ & 0.4 & $*$ & 0.8 & $*$ & 0.8 & 1.5 & $*$ & 1.5 & $*$ & 3.0 & 3.0 & $*$ & 6.0 & $*$ & 6.0 & $*$ & $*$ & 6.0 & $*$ & 6.0 & $*$ & 6.0 & 6.0 \\
\hline
\end{tabular}

\begin{tabular}{lccc} 
RESISTANCE FACTOR & & & \\
paclitaxel & 1.6 & 29.6 & 37.0 -fold \\
\cline { 2 - 2 } & 1.0 & & 0.3 -fold \\
\cline { 2 - 4 }
\end{tabular}

b
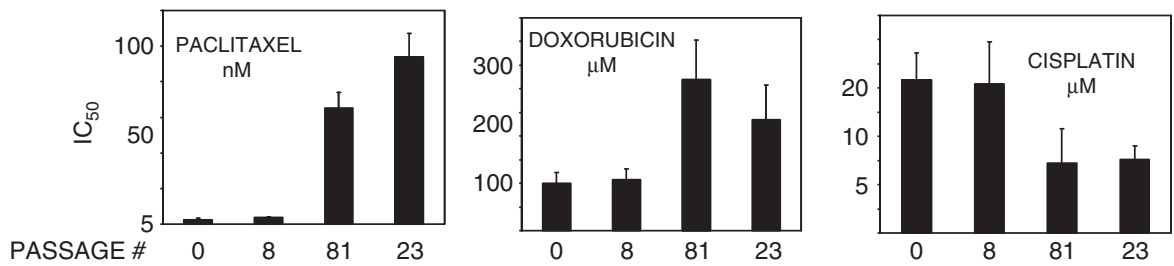

Figure 1 (a) Schema for development of paclitaxel-resistant cells in culture starting with parental MCF-7 cells at passage 1, week 1. RF indicates resistance factor, RF (i.e. $I C_{50}$ for resistant variant: $I C_{50}$ for parental cells. ${ }^{*}$ Indicates a drug-free passage to enable cells to recover from drug treatment. (b) Sensitivity to paclitaxel, doxorubicin and cisplatin in MCF7 cells with emerging resistance to paclitaxel. MCF-7TaxR cells with increasing resistance to paclitaxel were challenged with the indicated drugs and cytotoxicity determined using the MTT assay. Data shown are the mean $\mathrm{IC}_{50}$ and S.D. (shown by error bars) of at least four repeat analyses 
a

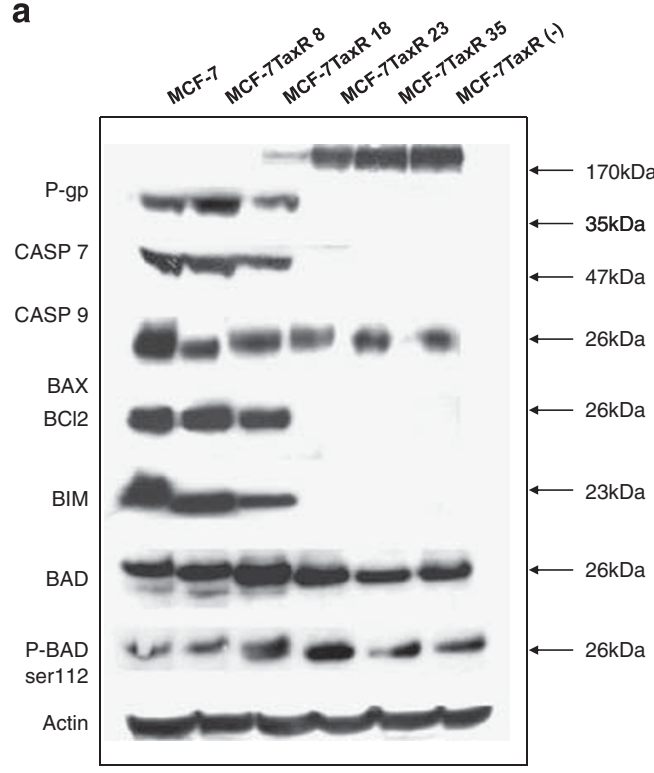

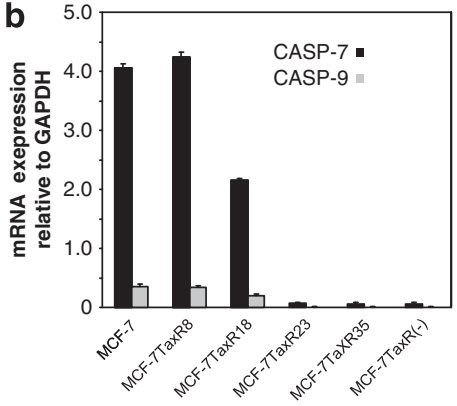

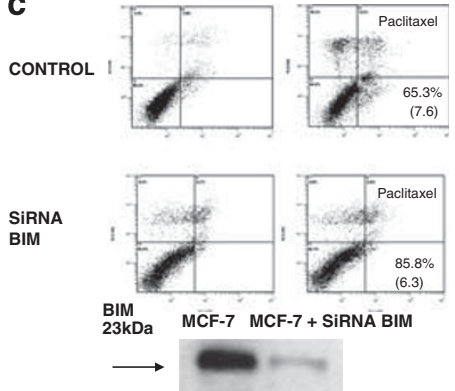

Figure 2 (a) Western immunoblotting of variant sublines of developing MCF-7TaxR lines. Whole-cell lysates were loaded onto SDS-PAGE gels (NOVEX, Invitrogen) at a concentration of $50 \mu \mathrm{g}$ and electrophoresis using Tris-Bis gels 10-12\% followed by transfer onto PVDF membranes. Primary antibodies to P-glycoprotein (P-gp), Bax, BCI2 were obtained from Santa Cruz, those for caspase-7 and 9, BAD and phosphorylated BAD and BIM were from Cell Signaling Technologies (distributed by New England Biolabs, Hitchin, UK) and then subjected to incubation with a secondary-alkaline phosphatase-conjugated antibody with subsequent chemiluminescent detection. All membranes were stripped and reprobed with an antibody to $\beta$-actin (Sigma Aldrich), $n=3$. (b) Quantitative PCR for assessment of mRNA levels of -7 and -9 on sublines of developing paclitaxel-resistant MCF-7 cells. Data shown are corrected for GAPDH expression using the equation $2^{-\Delta \Delta C_{t}}$. (c) Transfection of MCF-7 cells with SiRNA against BIM (Dharmacon) followed by drug treatment ( $20 \mathrm{nM}$ paclitaxel for $48 \mathrm{~h}$ ) subjected to flow cytometry using the Annexin $\mathrm{V}$ assay with PI. The percentage of live cells (expressed as a percentage of the untreated control) is indicated in the bottom left hand corner (PI and FITC negative cells) of the quadrant cytogram and data shown are representative of repeat data $(n=3)$. Comparisons of live populations of non-silenced cells versus BIM-silenced cells gave a $P$ value of 0.03 using a paired $t$-test. Assessment of efficiency of knockdown of BIM was made by western analysis (as described in Materials and Methods)

staurosporine treatment was of the order MDA-MB231 > MCF-7 > MCF-7TaxR. MDA-MB231 cells were chosen as a caspase-proficient line. ${ }^{6}$ Figure $3 \mathrm{C}$ shows the effects of cisplatin and paclitaxel following $48 \mathrm{~h}$ of continuous exposure. Treatment of cells with Z-VAD beyond $48 \mathrm{~h}$ is toxic and therefore, we could only use this treatment schedule. Moreover, treatment of MCF-7TaxR cells with a high dose of paclitaxel plus Z-VAD proved much more toxic than the same dose of paclitaxel alone. Hence, our data show the effects of $20 \mathrm{nM}$ paclitaxel, producing a loss of viability of approximately $25 \%$ in MCF-7 and only $8 \%$ loss of viability in the paclitaxel-resistant MCF-7TaxR line. Treatment of cells with cisplatin for $48 \mathrm{~h}$ shows more loss of viability for MCF-7 than for MCF-7TaxR. This is in contrast to the MTT data that show relative sensitivity of the MCF-7TaxR cells to the effects of platinum. It is important to note that a marked growth-inhibitory effect is seen with cisplatin and this will be measured by a lack of MTT reduction in the chemosensitivity testing, and this effect was pronounced in the MCF-7TaxR line.

Paclitaxel resistance is associated with diminished apoptotic response but upregulated autophagy. Treatment of MCF-7 and MCF-7TaxR cells with staurosporine with and without bafilomycin (an inhibitor of autophagic vesicle formation) then subjected to flow cytometric analysis with acridine orange, indicated an enhancement of acidic vesicle formation for the paclitaxel-resistant variant, which was shown to be statistically significantly different for MCF-7TaxR, based on $t$-test results (Figure 4a). Thus, treatment of MCF-7 and MCF-7TaxR lines with staurosporine resulted in a marked stimulation of autophagy for the latter. Inhibition of mTOR was noted for both MCF cell lines examined but more apparent for MCF-7TaxR cells, as seen in the disappearance of phosphorylated AKT, phophorylated mTOR and also the downstream substrate p60S6 kinase (p60S6-K), Figure 4b.

Figure 5 shows immunofluorescence for LC-3II using confocal microscopy, indicating the extent of autophagy present in staurosporine-treated breast cancer cell lines. For the caspase-proficient MDA-MB-231 cell line evidence of LC3II expression was not seen (Figure 5 a panel iv), whereas for MCF-7 presence of the LC-3II form in cells treated with $1 \mu \mathrm{M}$ staurosporine was seen, indicated by the heavy (green) staining (Figure $5 b$ panel iv). Figure $5 c$ shows the extent of LC3II formation for the established MCF-7TaxR line, panel iv, which is the heaviest staining seen for the three cell lines showing clear autophagosome vesicular formation, shown more clearly in Figure 5d.

Collateral cisplatin sensitivity is associated with mTOR inhibition. Using the autophagy inhibitors 3-methyl adenine and LY294002, we could see significant inhibition of cisplatin cytotoxicity, giving rise to increased $\mathrm{IC}_{50}$ values, as assessed using the MTT assay, Figure 6a. While the effects 
A

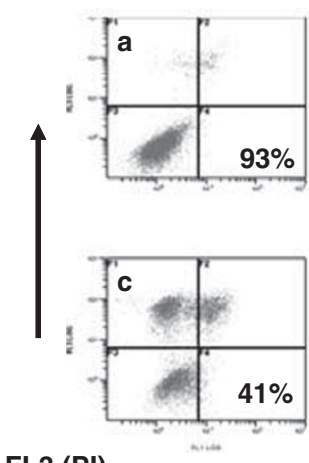

FL3 (PI)
MDA-MB-231

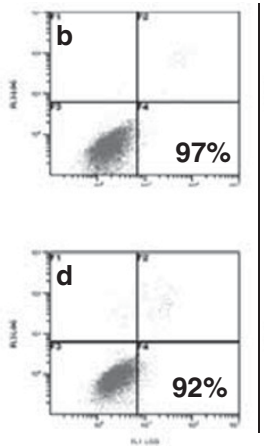

FL1 (FITC)

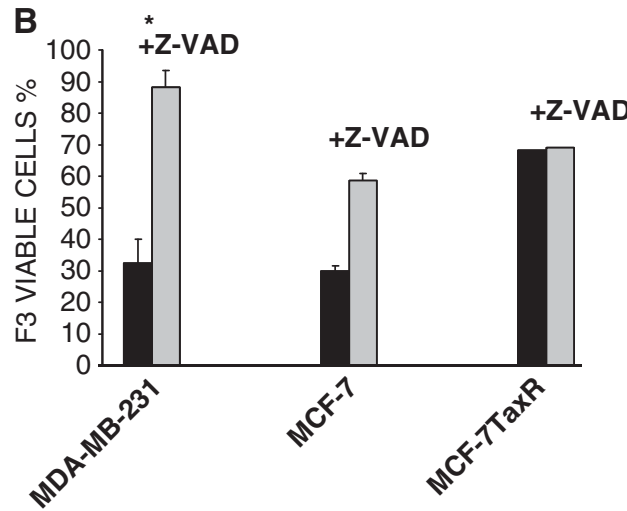

C

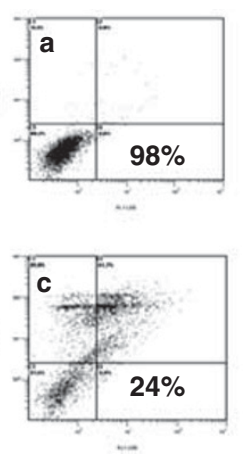

MCF-7
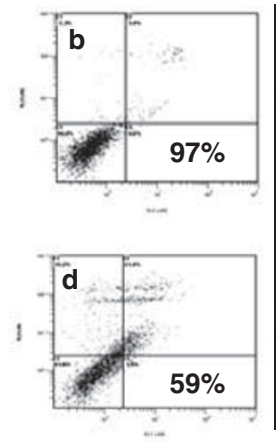

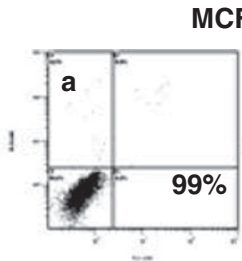

MCF-7TaxR

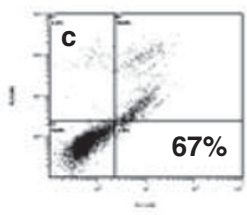

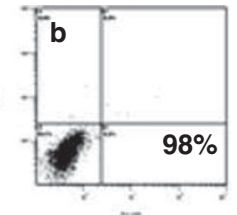

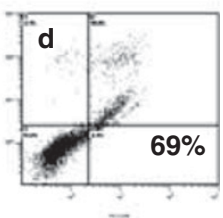

MCF-7

Cisplatin

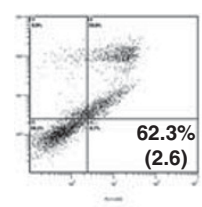
+ Z-VAD

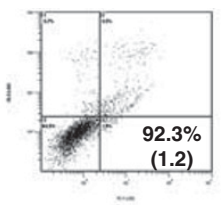

MCF-7TaxR
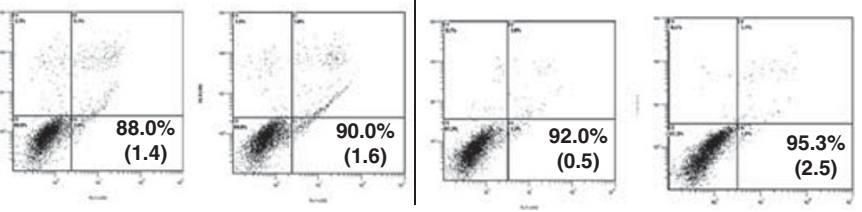

Figure 3 (A) Cytograms obtained from Annexin V assays of staurosporine $(1 \mu \mathrm{M})$-treated breast cancer cells. Each cytogram consists of data showing live cells (PI and FITC negative) bottom right; early apoptotic population (FITC positive) bottom right; mid-late stage apoptosis (PI and FITC positive) top right; necrotic/end stage apoptotic cells (PI positive, FITC negative) top left. $a=$ control untreated cells; $b=Z$ Z-VAD-treated control cells; $c=$ staurosporine-treated cells; $d=$ staurosporine and Z-VAD combination treatment. The live cell population is indicated for each sample and is representative of typical data obtained. (B) Bar graphs (derived from $\mathbf{A}$ ) showing extent of protection of staurosporine-treated cell population following $Z=V A D$ treatment. *Indicates $P$-value $<0.02$ (using a paired $t$-test). (C) Cytograms obtained for MCF-7 and MCF-7TaxR cells following $48 \mathrm{~h}$ continuous exposure to cisplatin $20 \mu \mathrm{M}$ in the absence and presence of Z-VAD and for paclitaxel $20 \mathrm{nM}$. Data shown are representative of repeat experiments and the numbers refer to the mean (and standard deviation) live (PI and FITC negative) populations (expressed as a percentage of the untreated controls) taken from $>3$ repeat experiments. $P$-values obtained for MCF-7 $\pm Z$-VAD with cisplatin $=0.003$ and the same figure obtained for MCF-7TaxR was $>0.5 ; P$-values obtained for MCF-7 $\pm Z$ Z-VAD with paclitaxel $=0.003$ and the same figure obtained for MCF-7TaxR was 0.5 (using a paired $t$-test)

of these two inhibitors were shown to be statistically significant in MCF-7 parental cells, the effects were more pronounced for the MCF-7TaxR cell line when treated with LY294002. These data provide evidence that the major cell death response found in MCF-7TaxR cells is autophagy.

\section{Upregulation of the autophagic pathway gives rise to} rapamycin resistance. MCF-7 and MCF-7TaxR cells were subjected to chemosensitivity testing with rapamycin and the $\mathrm{IC}_{50}$ values were shown to be $29.4 \mu \mathrm{M}( \pm 7.4)$ and $64.6 \mu \mathrm{M}$ $( \pm 9.6)$, respectively, Figure $6 \mathrm{~b}$. Using the mTOR inhibitor RAD001 (IC 50 95.5 nM-MCF-7; 150 nM-MCF-7TaxR), we could see a gradual reduction in the LC3II form following treatment with RAD001 $(100 \mathrm{nM})$ in MCF-7TaxR cells with increasing passage number, with the established resistant line showing MCF-7TaxR that shows negligible amounts of LC3II formation, Figure 6c. The survivin expression levels were markedly reduced in the MCF-7 parental cell line following treatment with RAD001, whereas for the MCF7TaxR variant sublines the expression levels of this IAP were consistently expressed with no variation following drug treatment. These data also indicate that as the survivin level in MCF-7 cells were markedly reduced with rapamycin treatment there is a possibility for some apoptotic induction, but this effect was not seen for the subvariants of the MCF paclitaxel-resistant lines. These particular data are consistent with activation of caspases for the MCF-7 parental line giving rise to activation of the mitochondrial pathway, whereas for the caspase-9-deficient MCF-7TaxR passage 18 and 23 variants and the established MCF-7TaxR line such an effect was not seen.

\section{Discussion}

We have derived paclitaxel-resistant MCF-7 human breast cancer cells using a drug exposure protocol similar to clinically used weekly paclitaxel regimens ( 3 weeks treatment followed by 1 week off for four cycles ${ }^{7}$ ) and employing drug doses well within the safety margins for clinical acceptable circulating levels of paclitaxel, that is, $<50 \mathrm{nM}$. We have used parental and paclitaxel-resistant cell lines to investigate changes in gene expression and cell death pathways that accompany paclitaxel resistance. Our data show that there are significant 


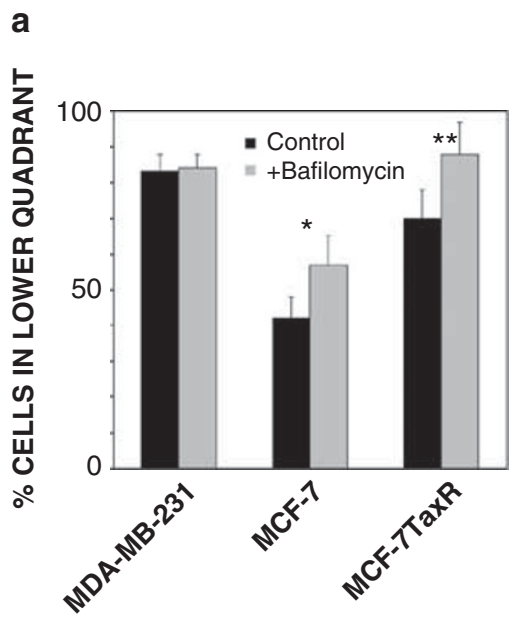

a b

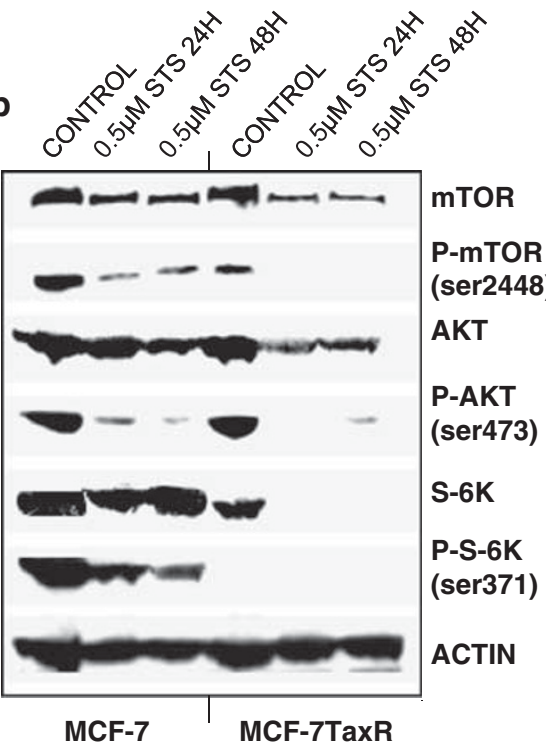

Figure 4 (a) Assessment of autophagic response of breast cancer cell lines to the effects of bafilomycin inhibition following treatment with $0.5 \mu \mathrm{M}$ staurosporine. Bar graphs represent the mean ( \pm S.D.) for $>3$ repeat experiments of the red fluorescence measured in the lower quadrant of the cytograms. Reduced values indicate migration to increased red and green fluorescence with formation of autophagic vesicles and reversal with bafilomycin. ${ }^{* \star} P=0.038,{ }^{* \star \star} P=0.012$ using paired Students $t$-test. (b) Western immunoblotting of MCF-7 parental and paclitaxel-resistant cells following treatment with staurosporine. Whole-cell lysates were made of cells following 24 and $48 \mathrm{~h}$ treatment and subjected to SDS-PAGE electrophoresis using NOVEX Bis-Tris gels (7-12\% or 10\%) and buffer system. Following transfer to PVDF membranes proteins were detected using the appropriate rabbit polyclonal antibody (Cell Signaling Technologies, obtained from New England Biolabs, Hitchin, UK) and then subjected to incubation with a secondary-alkaline phosphatase-conjugated antibody with subsequent chemiluminescent detection. All membranes were stripped and reprobed for actin as the loading control. Data shown are representative of three repeat experiments

a

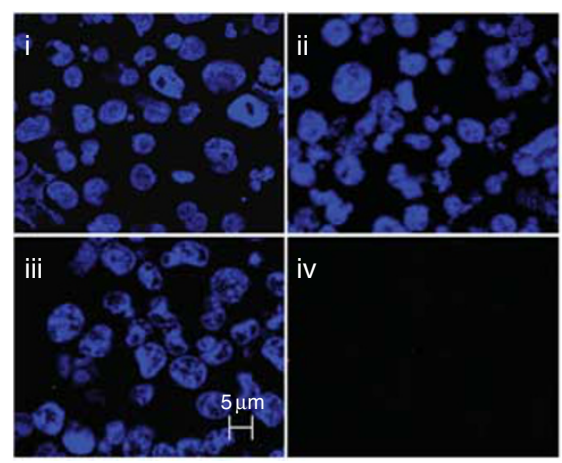

C
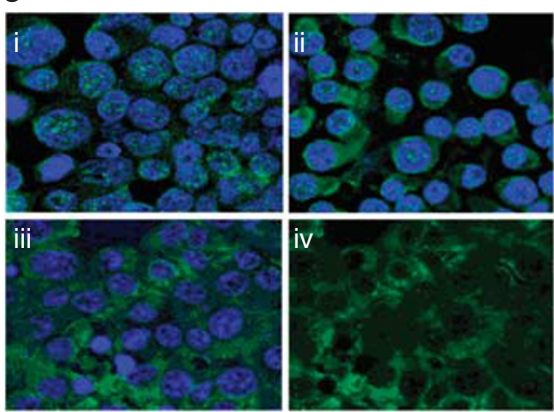

b

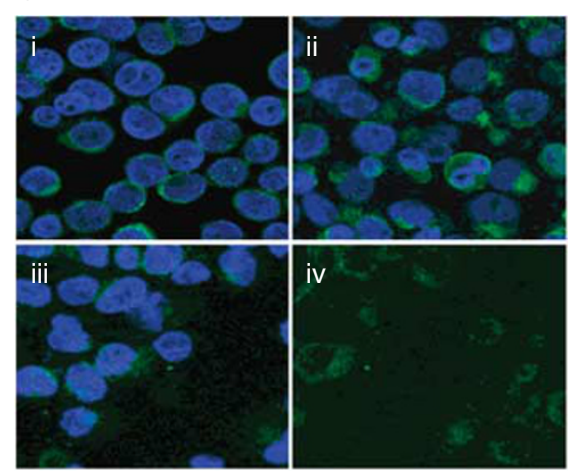

d

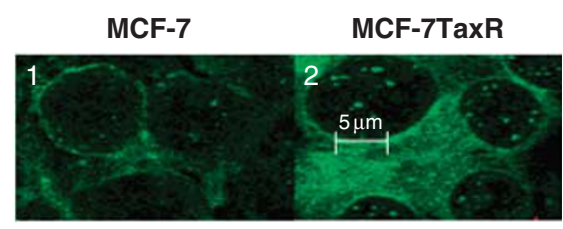

Figure 5 Confocal fluorescence microscopy imaging for LC-3 detection in MDA-MB-231, MCF-7 and MCF-7TaxR cell lines (a). Cells were incubated for $24 \mathrm{~h}$ with $0.25 \mu \mathrm{M}$ staurosporine (ii), or $0.5 \mu \mathrm{M}$ staurosporine (iii) and compared with untreated controls (i). Images (i-iii) were co-stained with the nuclear dye TOPRO-3 and images (iv) were stained for LC-3 alone (Alexa-Fluor 488 associated fluorescence). Images (d) (1) and (2) are enlarged images from (b) (iv) and (c) (iv) showing deposition of LC3ll granules in vacuoles in MCF-7 parental and MCF-7TaxR paclitaxel-resistant lines, respectively 

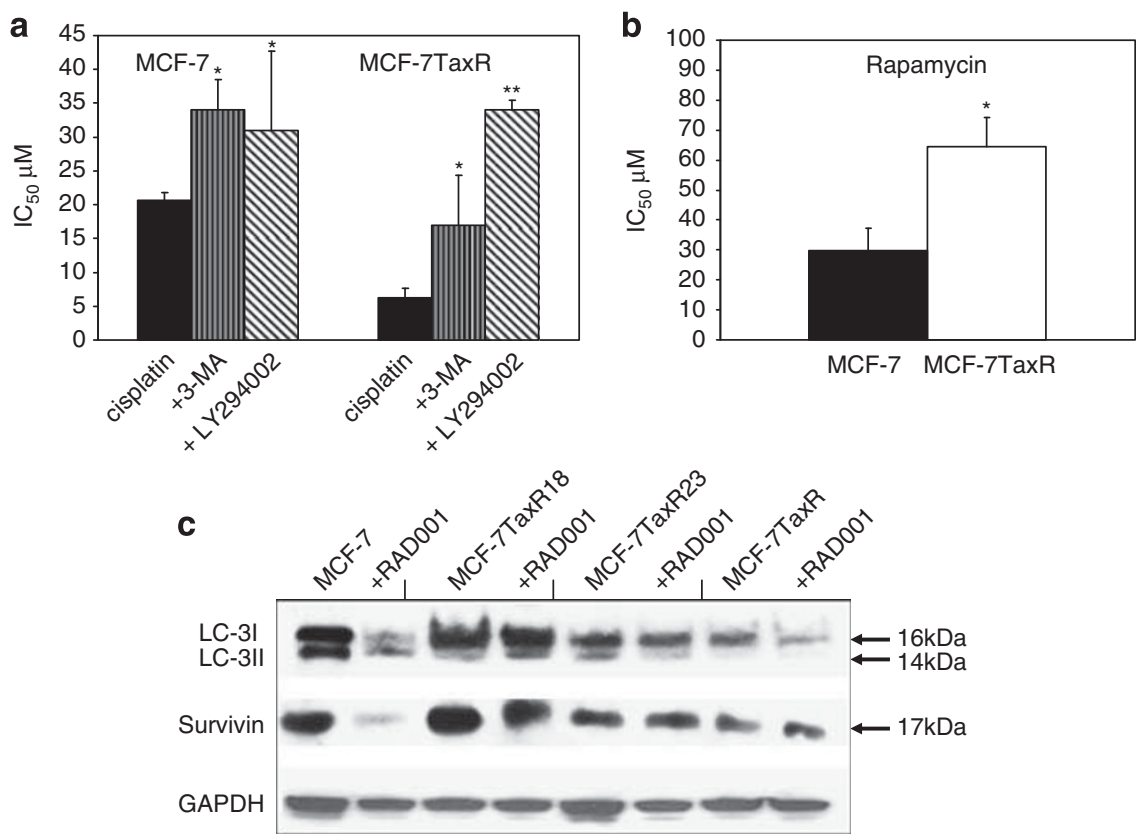

Figure 6 (a) Reduction in cisplatin sensitivity of breast cancer cells following co-treatment with autophagy inhibitors. Data shown are IC $\mathrm{C}_{50}$ values obtained using the MTT assay and represent the means and S.D. for $>3$ repeat experiments. $P$-values were calculated using the paired Student's $t$-test for $I_{50}$ cisplatin alone versus the $I C_{50}$ for combination of cisplatin with 3-methyladenine (3-MA) or LY294002. ${ }^{*} P<0.05,{ }^{* \star} P<0.02$. (b) IC 50 values obtained for MCF-7 cell lines treated with the mTOR inhibitor rapamaycin. *Denotes a $P$-value of $<0.02$ (using a paired Student's $t$-test). (c) Western immunoblotting of whole-cell lysate preparations of MCF-7 and paclitaxel-resistant sublines treated with RAD001 (100 nM) for $48 \mathrm{~h}$. Whole-cell lysates of control and drug-treated cultures were subjected to SDS-PAGE electrophoresis $10 \%$ Bis-Tris gels (NOVEX), western transfer onto PVDF membranes and probing with primary antibodies to LC-3I/II (Cell Signaling Technology), survivin (Santa Cruz). Membranes were then subjected to incubation with a secondary-alkaline phosphatase-conjugated antibody with subsequent chemiluminescent detection. All membranes were stripped and reprobed with an antibody to GAPDH (Sigma Aldrich), $n=3$

changes in expression of key regulators of cell death pathways and a striking switch from apoptosis to autophagy with increasing paclitaxel resistance.

A major finding in the present study was loss of expression of caspases-7 and -9 in MCF-7TaxR cells. As the first description of caspase-3 deficiency in MCF-7 cells with a 47-bp deletion in exon 3 of the CASP-3 gene ${ }^{8}$ an association of caspase deficiency with chemoresistance has been made. Interestingly, many breast cancer cell lines show weak expression of caspase- $3 .{ }^{6}$ Furthermore, it was reported that $75 \%$ of primary breast carcinomas have downregulation of caspase-3 mRNA, as seen in samples taken from breast cancer patients. ${ }^{9}$ Interestingly, peritumoral tissue also showed low expression of caspase-3, which suggests that loss of this caspase may be an important mechanism of survival for breast cancer cells. Those initial findings have been independently supported in studies reporting an association of caspase-3 deficiency with poor outcome in breast cancer. ${ }^{10}$ In the present study, we show deletion of caspases associated with the mitochondrial intrinsic pathway as a consequence of paclitaxel resistance in human breast cancer cells. We are not aware of previous reports citing loss of caspases-7 and -9 in association with taxane resistance. We are confident that the effects we are seeing are not relating any major mitochondrial dysfunction. As far as we can see the function of the mitochondria in the MCF-7TaxR is not impaired as we saw efficient reduction of MTT in the cytotoxicity tests used through out this study, and these data were corroborated by assessment of viability using trypan blue (not shown). In other studies we carried out we saw that caspase-8 expression and reactivity to anti-FAS was almost identical for the parental and paclitaxel-resistant MCF-7 cell lines alike, (data not shown). This led us to believe that the modulation of apoptosis we saw in the MCF-7TaxR line was predominantly relating to the intrinsic pathway.

A second important observation was loss of BIM expression in MCF-7TaxR. The BIM of cell death is a potent pro-apoptotic member of the Bcl2 family, ${ }^{11}$ essential for induction of cell death and to mediate cytochrome $c$ release from mitochondria which in turn activates apoptosome formation and the effector caspase-9. Here, we show using inhibitory RNA that BIM knockdown in parent MCF7 cells phenocopies MCF-7TaxR, implicating BIM as a key determinant of sensitivity to paclitaxel. Consistent with the present results in MCF-7 cells, knockdown of BIM reduced paclitaxel cytotoxicity in neuroblastoma cells but failed to protect against etoposide or cisplatin-induced death. ${ }^{12}$ In partial agreement with this, in our drug-resistant cell line MCF-7TaxR loss of BIM expression was accompanied by a collateral increase in sensitivity to cisplatin. Interestingly, we earlier showed that the sensitivity of P-glycoprotein-expressing MCF-7TaxR cells to etoposide was unchanged compared with the parental cell line. ${ }^{4}$ Previously, upregulation of P-glycoprotein has been reported to result in resistance to etoposide. ${ }^{13}$ As such, our data obtained for MCF7TaxR are somewhat unusual and imply that upregulation of P-glycoprotein per se is insufficient to mediate etoposide resistance. It is also interesting to note that at the point when resistance to paclitaxel became more marked in the 
developing MCF-7TaxR cells, this coincided with the disappearance of BIM (passage \#18).

Perhaps the most striking observation from our studies is the switch from apoptosis to autophagy as the major mechanism of cell death in MCF-7TaxR cells. A study which looked at the autophagic response of ovarian cancer cells treated with RAD001 reported that cisplatin-resistant cell lines were significantly more sensitive, supports our findings. ${ }^{14}$ Cisplatin-sensitive MCF-7TaxR cells were markedly more resistant to rapamycin, in line with a reciprocal relationship for the sensitivity of cancer cells to these two agents. The emergence of increasing sensitivity to platinum agents is of particular interest and is relevant to the management of breast and ovarian cancers. The data describing sensitivity of MCF-7 cells to RAD001 are in close agreement with a report by Treck et al. ${ }^{15}$ and other data we show indicates that when autophagy survival fails in the MCF-7TaxR line then death occurs predominantly via autophagy. A study which looked at the autophagic response of cisplatin- sensitive and -resistant clear cell ovarian cancer cells when treated with RAD001 is in some agreement with our current study (Mabuchi et al. ${ }^{14}$ ), which reported that cisplatin-resistant cell lines were shown to be significantly more sensitive to RAD001. In the current study, cisplatin-sensitive MCF-7TaxR cells were shown to be significantly more resistant to rapamycin, suggesting a reciprocal relationship for the sensitivity of cancer cells to these two agents.

AKT has previously been described as an important determinant of chemosensitivity for many kinds of cells. ${ }^{14}$ Currently, we have shown that there was little evidence of phospho-mTOR expression and no evidence for activation of AKT when MCF-7TaxR cells were treated with staurosporine, in line with an enhanced autophagic response, with modest but nevertheless higher expression levels of these proteins seen for parental MCF-7 cells. A report by Faried et al. ${ }^{16}$ described significant reductions in AKT induced following paclitaxel treatment but increases in phosphorylated mTOR in cervical cancer cells. Further, the same report described a lack of correlation between AKT levels and paclitaxel sensitivity but a closer correlation was seen with mTOR levels. RAD001 can effectively inhibit mTOR signalling and this resulted in a marked reduction in phosphorylated p70S6K with little effect on phospho-AKT expression in ovarian cancer cells. ${ }^{14}$ In agreement with this, we showed that staurosporinetreated MCF-7TaxR cells markedly attenuated levels of the mTOR substrate phospho-p70S6K. Confirmation of the marked autophagic response elicited in staurosporine-treated MCF-7TaxR cells was seen in confocal images, where marked LC3II formation was seen in MCF-7TaxR cells. In contrast, rapamycin-resistant MCF-7TaxR variant cell lines failed to undergo autophagic cell death following treatment with RAD001. When the various paclitaxel-resistant sublines of MCF-7 were subjected to treatment with RAD001, we saw no evidence of formation of LC-3II, in line with the relative resistance of these cells to the drug, compared with the parental line. The disappearance of survivin in MCF-7-treated cells is in some agreement with Kang et al. ${ }^{17}$ who described a cross talk between autophagy and apoptosis, wherein degradation of survivin is seen via a beclin-mediated pathway. As both pathways are operative for MCF-7 cells this effect was seen, but where there was an absence of functioning apoptosis then survivin levels remained constant.

In order to explain the effects, we have seen in the developing paclitaxel resistance of MCF-7 cells there are a number of factors we can consider. A polyploidy state has been described in resistance to paclitaxel ${ }^{18}$ and indeed there are many genes that are implicated in taxane resistance that have roles in the maintenance of chromosomal stability. ${ }^{19,20}$ Thus, paclitaxel is deemed to be more effective in cell populations that are chromosomally stable, whereas therapies such as trastuzumab have activity in more unstable cell populations. ${ }^{21}$ An aneuploid state with a high number of DNA breakpoints has been associated with preferential sensitivity to platinum agents in breast, ovarian and gastric cancers. ${ }^{21}$

Finally, our data highlight the very important observation that the absence of an apoptotic response results in abolition of an MDR phenotype in the presence of P-glycoprotein upregulation. A report by Johnstone et al. ${ }^{22}$ described the protection of leukaemic cells by the presence of upregulated $\mathrm{P}$-glycoprotein against caspase-dependent but not caspaseindependent cell death. This report corroborates our finding that caspase-deficient or caspase-inactivated, P-glycoprotein expressing paclitaxel-resistant breast cancer cells do not show cross-resistance with doxorubicin, colchicine or etoposide, as shown by the present study and previous work by us. ${ }^{4}$ We did see, however, that the MDR-reversing agent Tariquidar (XR9576) was able to reverse paclitaxel resistance in the MCF-7TaxR line, whereas the sensitivity to the other MDR-associated agents was unchanged. The basis for this agent-specific effect is currently unclear and highlights the complexity of the MDR phenotype.

In conclusion, we have shown that development of resistance to the commonly used chemotherapeutic agent paclitaxel gives rise to significant molecular changes that culiminate in a caspase-deficient genotype dictating a predominantly autophagic response to chemotherapeutic agents. Our data also suggest that in the absence of multiple caspases and also of several BCL2 family members marked attenuation of MDR takes place. These findings have implications for breast cancer treatment where low expression levels of caspases may be prevalent.

\section{Materials and Methods}

Chemicals and reagents. Reagents were obtained from Sigma Aldrich (Poole, UK) unless stated otherwise. Staurosporine (Alexis Biochemicals, Nottingham, UK), RAD001 (Selleck Chemicals, Houston, TX, USA), Z-VAD (Bachem, Bubendorf, Switzerland), 3-methyl-adenine, LY294002, bafilomycin and rapamycin were dissolved in dimethyl sulphoxide and stored as stock solutions; doxorubicin was made up in sterile distilled water. Paclitaxel (Taxol; obtained from Bristol Myers Squibb, Uxbridge, UK, as a pharmacy preparation) and cisplatin were diluted in sterile $0.9 \%$ saline as stock solutions stored at $-20^{\circ} \mathrm{C}$ until use.

Cell culture. The human breast cancer cell line MDA-MB-231 (ATCC from LGC Promochem, Teddington, UK) was cultured in L15-Leibovitz medium (Sigma Aldrich). The human breast carcinoma MCF-7 cell line was obtained from the European Collection of Cell Cultures (ECACC; Porton Down, Salisbury, UK) and maintained at a low passage number throughout the study. Cell lines were authenticated at source by STR profiling, morphology (ATCC) and DNA profiling (ECACC). MCF-7 sublines were cultured in Dulbecco's modified Eagle's (Sigma Aldrich) media with $2 \mathrm{mM}$ of Glutamax and supplemented with $10 \%$ heat-inactivated foetal bovine serum (Invitrogen, Paisley, UK) at $5 \% \mathrm{CO}_{2} / 37^{\circ} \mathrm{C}$. Cell lines with acquired resistance to paclitaxel were generated by growth in incrementally 
increasing concentrations with successive passages (Figure 1). The resulting MCF-7 cell line with paclitaxel resistance, designated MCF-7TaxR, was maintained at $6 \mathrm{nM}$ paclitaxel with treatment at alternate passages. The established MCF-7TaxR cell line was designated as those cultures with passage number exceeding 23 . Withdrawal of paclitaxel from the culture medium in MCF-7TaxR cells at passage 40 with subsequent culture as for parental cells over several weeks ( $>9$ months) resulted in the cell variant MCF-7TaxR(-). This subline was developed to examine the stability of the drug-resistant phenotype. All cell lines were grown in drug-free media for at least one passage before experimental use. Mycoplasma testing was carried out regularly using a polymerase chain reaction (PCR) -based methodology.

Cytotoxicity testing. Human breast cancer cells MCF-7, MCF-7TaxR passage 8 , MCF-7TaxR passage 18 and MCF-7TaxR passage 23 were grown in 96-well plates at a density of $3 \times 10^{4} / \mathrm{ml}$ for $24 \mathrm{~h}$. Drugs were diluted in supplemented tissue culture medium and added in increasing concentration, with untreated wells as controls. Cells were then incubated for a period of $96 \mathrm{~h}$ and subjected to the 3-(4,5-dimethylthylthiazol-2-yl)-2,5-diphenyltetrazolium bromide (MTT) assay. This incubation period accounted for the doubling times of the cell lines (which did not vary significantly between parental and resistant variants) and allowed for an approximate 10-20-fold increase in cell number of control cells during this time period. For treatment with rapamycin and RAD001 cells were subjected to staining with trypan blue and cell counting used as a measure of cell viability. This method was also used to confirm the MTT data previously obtained that indicated paclitaxel resistance for MCF-7TaxR cells, set up alongside parental MCF-7 cells for comparison. $\mathrm{IC}_{50}$ values (calculated as the dose necessary to cause a $50 \%$ reduction in cell viability relative to untreated control cells) were calculated from dose response curves using PRISM software.

Annexin $\mathrm{V}$ with propidium iodide (PI) methodology for apoptosis with flow cytometry. Cells were seeded into tissue culture flasks to give approximately $50 \%$ confluence, allowed to attach for $2-3 \mathrm{~h}$ and then treated for a period of 24-48 $\mathrm{h}$ with the appropriate compound at various concentrations (see figure legends). Cells for treatment with the pan-caspase inhibitor Z-VAD (100 $\mu \mathrm{M})$ were allowed to settle and then treated for $1 \mathrm{~h}$ before treatment with staurosporine $(1 \mu \mathrm{M})$ for $24 \mathrm{~h}$ or with paclitaxel $(20 \mathrm{nM})$ or cisplatin $(20 \mu \mathrm{M})$ for a period of $48 \mathrm{~h}$. An Annexin V-fluoroscein-isothiocyanate (FITC)-conjugated apoptosis detection kit incorporating PI was used as described by the manufacturer's protocol (Oncogene; supplied by CN Biosciences, Beeston, UK). Harvesting of cells included collection of attached cells following trypsinisation and floating cells, which were combined in the sample for processing. Analysis by flow cytometry used the FL1 (FITC) and FL3 (PI) laser lines and each sample was assessed using a collection of 10000 events.

Western blotting analysis. Whole-cell lysates were made from monolayers of cultured cell in the exponential phase of growth. Cells were subjected to various treatment protocols (as indicated in the figure legends) after seeding into tissue culture flasks at $30-40 \%$ confluence and left to equilibrate for $24 \mathrm{~h}$. Cells were harvested by scraping or by the use of a non-enzymatic cell dissociation fluid, washed twice in PBS and then resuspended in hypotonic lysis buffer containing detergent and protease inhibitors. A $50-\mu \mathrm{g}$ of membrane protein was loaded onto SDS-PAGE gels and electrophoresed (Novex system, Invitrogen), and western transferred to a polyvinylidene difluoride membrane. Probing of the membranes for $\beta$-actin or glyceraldehyde phosphate dehydrogenase (GAPDH) levels to act as loading controls was carried out by membrane stripping with $1 \mathrm{mM}$ of sodium azide in PBS for $1 \mathrm{~h}$ followed by incubation using murine antibody to actin or GAPDH (Sigma Aldrich). Visualisation was carried out using anti-rabbit or anti-mouse alkaline phosphatase-conjugated secondary antibody (WesternBreeze) with chemiluminescent substrate (CDP Star) both obtained from Invitrogen.

Qualitative PCR analysis. RNA was extracted from monolayers of human breast cancer cell lines using Trizol reagent (Invitrogen) according to the manufacturer's instructions. Quantitiave PCR analysis was carried out using SYBRgreen reagent (One-step QuantiTect Qiagen, Crawley, UK). Primers for caspases-7 and -9 were from SuperArray (obtained from Tebu-Bio, Leicester, UK). The PCR reactions were carried out using the TaqMan 700 ABI Prism with software from Applied Biosystems (Life Technologies, Paisley, UK), setting the cycles as follows: $30 \mathrm{~min} / 50^{\circ} \mathrm{C}$ for reverse transcription; $15 \mathrm{~min} / 95^{\circ} \mathrm{C}$ PCR initial activation step; 40 cycles of denaturation for $20 \mathrm{~s} / 95^{\circ} \mathrm{C}$ and annealing step for $60 \mathrm{~s} / 60^{\circ} \mathrm{C}$. Fold changes were calculated using the formula $2^{\left(-\Delta \Delta C_{t}\right)}$, where $\Delta \Delta C_{t}$ is the $\Delta C_{\mathrm{t}(\text { gene of interest) }}-\Delta C_{\mathrm{t}(\mathrm{GAPDH})}$ and $C_{\mathrm{t}}$ is the cycle at which the threshold is crossed.
Transfection with $\mathrm{BCl}$-interacting mediator of cell death (BIM) small interfering RNA (SiRNA). MCF-7 cells were subjected to transfection with SiRNA to BIM (Dharmacon; distributed by ThermoScientific/AbGene Ltd, Epsom, UK) reagent SiGENOME (product code D-004383-20), target sequence: 5'-GUUCUGAGUGUGACCGAGA-3', using transfection reagent DharmaFECT1 (product code T-2001-02) according to manufacturer's instructions with OPTIMEM medium (Invitrogen). The appropriate negative controls were used according to the manufacturer's protocols.

Acridine orange staining for detection of acidic vesicles. In order to detect and quantify the acidic vesicle formation during the process of autophagy, MCF-7 cell lines were exposed to $1 \mu \mathrm{M}$ staurosporine treatment for $24 \mathrm{~h}$. Cells were then harvested by trypsinisation. Cells were washed twice with PBS and then stained with acridine orange solution (Sigma Aldrich) at a final concentration of $2 \mathrm{ng} / \mathrm{ml}$ for $30 \mathrm{~min}$ in the dark. Acridine orange is a weak base that can accumulate in acidic spaces and emits bright red fluorescence (measured at FL4). The intensity of the red fluorescence is proportional to the degree of acidity and/or volume of the cellular acidic compartments. Bafilomycin treatment is used to inhibit the autophagosome formation. The extent of autophagy (as a \% on the FL4 scale) is thus assessed as the extent of red fluorescence minus that seen in the presence of bafilomycin.

Fluorescence confocal microscopy for detection of LC3. Cells were seeded into T25 $\mathrm{cm}^{2}$ tissue culture flasks at densities of $2 \times 10^{5}$ (MDA-MB-231) or $3 \times 10^{5}$ (MCF-7, MCF-7TaxR) and left for $24 \mathrm{~h}$ under standard culture conditions. Cells were then treated with a sub-cytotoxic dose of staurosporine at a dose of 0.25 or $0.5 \mu \mathrm{M}$ for a further $24 \mathrm{~h}$. Cells were harvested using standard trypsinisation with collection of the floating cell population and processed using the Cytospin 4 cytological centrifuge (ThermoElectron Corporation, Basingstoke, UK). The cytospin slides were then fixed in 2-4\% paraformaldehyde/PBS for $15 \mathrm{~min}$ and rinsed in PBS. Slides were then covered in ice-cold methanol and kept at $-20^{\circ} \mathrm{C}$ for $10 \mathrm{~min}$ (permeabilisation stage). Following PBS rinsing slides were blocked with $5 \%$ goat serum for non-specific binding for $1 \mathrm{~h}$. This was then followed by overnight incubation with primary antibody (LC-3B; Cell Signaling Technology) at a dilution of 1:200 in PBS-Triton, in a humidifying chamber at $4^{\circ} \mathrm{C}$. Slides were then washed with PBS and incubated with the secondary antibody conjugated to Alexa-Fluor488 anti-rabbit secondary antibody (Molecular Probes, Invitrogen) for $2 \mathrm{~h}$. After rinsing with PBS then the nuclear dye TOPRO3 (Nuclear Probes, Invitrogen) was applied for 10 min with a final washing using high-salt PBS. Slides were left to air-dry and then Anti-Fade reagent for mounting was used on a coverslip. The edges of the slides were then sealed with standard commercially available nail lacquer. Images were produced by confocal microscopy using a Carl Zeiss Laser Scanning System, LSM510 at $\times 40$ magnification.

\section{Conflict of Interest}

The authors declare no conflict of interest.

1. Bergamaschi D, Samuels Y, O'Neill NJ, Trigiante G, Crook T, Hsieh JK et al. iASPP oncoprotein is a key inhibitor of p53 conserved from worm to human. Nat Genet 2003; 33 162-167.

2. Okada H, Mak TW. Pathways of apoptotic and non-apoptotic death in tumour cells. Nat Rev Cancer 2004; 4: 592-603. (review).

3. Mashima T, Tsuruo T. Defects of the apoptotic pathway as therapeutic target against cancer. Drug Resist Updat 2005; 8: 339-343. (review).

4. Coley HM, Labeed FH, Thomas H, Hughes MP. Biophysical characterization of MDR breast cancer cell lines reveals the cytoplasm is critical in determining drug sensitivity. Biochimica et Biophysica Acta 2007; 1770: 601-608.

5. Seve $\mathrm{P}$, Dumontet $\mathrm{C}$. Is class III $\beta$-tubulin a predictive factor in patients receiving tubulinbinding agents? Lancet Oncol 2008; 9: 168-175.

6. Yang S, Liu J, Thor AD, Yang X. Caspase expression profile and functional activity in a panel of breast cancer cell lines. Oncol Rep 2007; 17: 1229-1235.

7. Qi M, Li JF, Xie YT, Lu AP, Lin BY, Ouyang T. Weekly paclitaxel improved pathologic response of primary chemotherapy compared with standard 3 weeks schedule in primary breast cancer. Breast Cancer Res Treat 2010; 123: 197-202.

8. Janicke RU, Ng P, Sprengart ML, Porter AG. Caspase-3 is required for alpha-fodrin cleavage but dispensible for cleavage of other death substrates in apoptosis. J Biol Chem 1998; 273: 15540-15545

9. Devarajan E, Sahin AA, Chen JS, Krishnamurthy RR, Aggarwal N, Brun AM et al. Downregulation of caspase-3 in breast cancer: a possible mechanism for chemoresistance. Oncogene 2002; 21: 8843-8851. 
10. Nassar A, Lawson D, Cotsonis G, Cohen $C$. Survivin and caspase-3 expression in breast cancer: correlation with prognostic parameters, proliferation, angiogenesis, and outcome. Appl Immunohistochem Mol Morphol 2008; 16: 113-120.

11. Gillings AS, Balmanno K, Wiggins CM, Johnson M, Cook SJ. Apoptosis and autophagy: $\mathrm{BIM}$ as a mediator of tumour cell death in response to oncogene-targeted therapeutics. FEBS J 2009; 276: 6050-6062.

12. Li Z, Zhang J, Liu Z, Woo CW, Thiele CJ. Downregulation of Bim by brain-derived neurotrophic factor activation of TrkB protects neuroblastoma cells from paclitaxel but not etoposide or cisplatin-induced cell death. Cell Death Differ 2007; 14: 318-326.

13. Konno T, Ebihara T, Hisaeda K, Uchiumi T, Nakamura T, Shirakusa T et al. Identification of domains participating in the substrate specificity and subcellular localization of the multidrug resistance proteins MRP1 and MRP2. J Biol Chem 2003; 278: 22908-22917.

14. Mabuchi S, Kawase C, Altomare DA, Morishige K, Sawada K, Hayashi M et al. mTOR is a promising therapeutic target both in cisplatin-sensitive and resistant clear cell carcinoma of the ovary. Cancer Res 2009; 15: 5404-5413.

15. Treeck O, Wackwitz B, Haus U, Ortmann O. Effects of a combined treatment with mTOR inhibitor RAD001 and tamoxifen in vitro on growth and apoptosis of human cancer cells. Gynecol Oncol 2006; 102: 292-299.

16. Faried LS, Faried A, Kanuma T, Nakazato T, Tamura T, Kuwano $\mathrm{H}$ et al. Inhibition of mammalian target of rapamycin (mTOR) increases chemosensitivity of CaSki cells to paclitaxel. Eur J Cancer 2006; 42: 934-947.

17. Kang R, Zeh HJ, Lotze MT, Tang D. The beclin 1 network regulates autophagy and apoptosis. Cell Death Differ 2011; 18: 571-580.
18. Bouchet BP, Bertholon J, Falette N, Audoynaud C, Lamblot C, Puisieux A et al. Paclitaxel resistance in untransformed human mammary epithelial cells is associated with an aneuploidy-prone phenotype. $\mathrm{Br} J$ Cancer 2007; 97: 1218-1224.

19. Pusztai L. Markers predicting clinical benefit in breast cancer from microtubule-targeting agents. Ann Oncol 2007; 18: 15-20. (review).

20. Sudo T, Nitta M, Saya H, Ueno NT. Dependence of paclitaxel sensitivity on a functional spindle assembly checkpoint. Cancer Res 2004; 64: 2502-2508.

21. Burrell RA, Juul N, Johnston SR, Reis-Filho JS, Szallasi Z, Swanton C. Targeting chromosomal instability and tumour heterogeneity in HER2-positive breast cancer. J Cell Biochem 2010; 111: 782-790.

22. Johnstone RW, Cretney E, Smyth MJ. P-glycoprotein protects leukaemia cells against caspase-dependent but not caspase-independent cell death. Blood 1999; 93: 1075-1085.

Cell Death and Disease is an open-access journal published by Nature Publishing Group. This work is licensed under the Creative Commons Attribution-Noncommercial-No Derivative Works 3.0 Unported License. To view a copy of this license, visit http://creativecommons.org/licenses/by-nc-nd/3.0/ 